\title{
Superposition operators and the order and type of entire functions
}

\author{
Stephen M. Buckley and Dragan Vukotić
}

\begin{abstract}
We distinguish between entire functions of different order or type by the behavior of their associated superposition operators on subsets of Besov spaces or the little Bloch space.
\end{abstract}

\section{Introduction}

Let $X, Y$ be spaces of analytic functions on the unit disk $\mathbb{D} \subset \mathbb{C}$ which contain the polynomials. The nonlinear superposition operator $S_{\varphi}$ on $X$ (with symbol $\varphi$ ) is defined by

$$
S_{\varphi}(f)=\varphi \circ f, \quad f \in X .
$$

We write $\varphi \in S(X, Y)$ if $S_{\varphi}(X) \subset Y$. It is immediate that $S(X, Y)$ is a vector space of entire functions. The following natural questions arise:

(1) Given spaces $X, Y$, what is $S(X, Y)$ ?

(2) Are there $X, Y$ for which $S(X, Y)$ equals a given space of entire functions? Similar problems in the context of real variables have a long history $[\mathbf{A Z}]$, but the above questions have only recently been studied in complex function theory. Cámera and Giménez answered (1) in the context of Bergman spaces and the Nevanlinna area class $[\mathbf{C G}]$ and Hardy spaces and related classes $[\mathbf{C a}]$. Together with J.L. Fernández $[\mathbf{B F V}]$, we answered (1) for various classes of Dirichlet-type spaces. Question (2) is most natural for spaces of entire functions $\varphi$ defined in terms of a bound on the growth rate of $\varphi$, and we showed in $[\mathbf{B F V}]$ that many such spaces of entire functions were of the form $S(X, Y)$ for pairs of appropriate Dirichlet-type spaces $X, Y$. More precisely, we showed that this the case for the space of polynomials of degree at most $n \geq 0$ (see Corollary 10 and Theorem 12) and the spaces $E(\rho)$ for each $\rho>1$ (see Corollary 25); here $E(\rho)$ is the space of functions of order less than $\rho$, or order $\rho$ and finite type. Other related results on superposition operators from Bloch or Besov space to Bergman space were proved in $[\mathbf{A M V}]$ and $[\mathbf{B V}]$.

These earlier results involving $E(\rho)$ can be viewed as "tests" that distinguish between entire functions of different orders according to whether or not they map

2000 Mathematics Subject Classification. 47H30, 31C25, $30 \mathrm{H} 05$.

This research of both authors was supported by MCyT grant BFM2003-07294-C02-01, Spain. The first author was also supported by Enterprise Ireland. The second author was also partially supported by MTM2004-21420-E (Acciones Especiales), MEC, Spain. 
Dirichlet-type spaces to other Dirichlet-type or Bergman spaces. However none of these earlier results provide us with tests to distinguish between entire functions of the same order but different types. It is precisely this refinement that we examine in this note. In particular we show that superposition operators from Besov spaces $X=B^{p}$ or the little Bloch space $X=\mathcal{B}_{0}$ to Bergman spaces $Y=A^{q}$ can be used to distinguish between entire functions that have the same order $\rho \geq 1$ but different types. This distinction requires a more refined criterion than simply whether the operator $S_{\varphi}$ maps all of one space into another. Instead the test will be whether $S_{\varphi}(f)$ lies in a fixed ball in $Y$ for all $f$ in some bounded subset of $X$.

Let us state here one such result which implies that superposition operators from Dirichlet space $\mathcal{D}=B^{2}$ to Bergman space $A^{q}$ can be used in order to distinguish between entire functions of order 2 and distinct types. For a definition of the space $E(2, \tau)$, see Section 2 ; for now, we remark that $E(2, \tau)$ contains all functions whose order and type strictly precede $(2, \tau)$ lexicographically.

TheOREM 1.1. Suppose $0<\tau, q<\infty$. Let $R:=\sqrt{2 / \tau q}$ and $K_{R}:=\{f \in \mathcal{D}$ : $\left.f(0)=0,\|f\|_{\mathcal{D}} \leq R\right\}$. Then $\varphi \in E(2, \tau)$ if and only if $S_{\varphi}\left(K_{R}\right)$ is a bounded set in $A^{q}$.

\section{Background}

Throughout the paper, $\mathbb{C}$ denotes the complex plane, $\mathbb{D}$ the unit disk $\{z \in \mathbb{C}$ : $|z|<1\}, \mathbb{T}$ the unit circle $\{z \in \mathbb{C}:|z|=1\}$, and $d A(z):=\pi^{-1} d x d y=\pi^{-1} r d r d \theta$ is normalized area measure (a probability measure) on $\mathbb{D}$. A disk of radius $r$ centered at $w$ is denoted $D(w, r)$, while $[z, w]$ means the line segment from the point $z$ to the point $w$.

Given two positive expressions $A, B$, we write $A \lesssim B$ or $B \gtrsim A$ to mean that $A \leq C B$ for some constant $C$ dependent only on allowed parameters (which should be clear from the context). We write $C=C(A, B, \ldots)$ to indicate that $C$ depends only on the parameters $A, B, \ldots$.

2.1. Order and type. Recall that the order of a non-constant entire function $\varphi$ is

$$
\rho:=\limsup _{r \rightarrow \infty} \frac{\log \log M(r, \varphi)}{\log r},
$$

where $M(r, \varphi):=\max \{|\varphi(z)|:|z|=r\}$. If $\varphi$ has order $\rho<\infty$, then the type $\tau$ of $\varphi$ is

$$
\tau:=\limsup _{r \rightarrow \infty} \frac{\log M(r, \varphi)}{r^{\rho}} .
$$

See the first two chapters of [Boa] for more on entire functions. The allowable (order-type) pairs are $(\rho, \tau), 0 \leq \rho<\infty, 0 \leq \tau \leq \infty$, together with the special pair $(\infty, *)$, where $*$ just means that type is undefined for infinite order functions. Let us define $\prec$ on this set of allowable pairs to be the (strict) lexicographic order. Thus $\left(\rho_{1}, \tau_{1}\right) \prec\left(\rho_{2}, \tau_{2}\right)$ if either $\rho_{1}<\rho_{2} \leq \infty$ or $\rho_{1}=\rho_{2}<\infty$ and $\tau_{1}<\tau_{2}$.

Suppose $(\rho, \tau)$ is an allowable pair with $\rho<\infty$. We denote by $E^{\prime}(\rho, \tau)$ the space of entire functions that grow more slowly than some entire function of order $\rho$ and type $\tau$. Equivalently, $\varphi \in E^{\prime}(\rho, \tau)$ if $\varphi$ is constant, has order less than $\rho$, or has order $\rho$ and type at most $\tau$. If $\tau<\infty$, we also define $E(\rho, \tau)$ to be the space of entire functions $\varphi$ for which there exists a constant $C$ such that $|\varphi(z)| \leq C \exp \left(\tau|z|^{\rho}\right)$, $z \in \mathbb{C}$. Note that $E^{\prime}\left(\rho^{\prime}, \tau^{\prime}\right) \subset E(\rho, \tau) \subset E^{\prime}(\rho, \tau)$ whenever $\left(\rho^{\prime}, \tau^{\prime}\right) \prec(\rho, \tau)$. Thus 
there is a close relationship between $E(\rho, \tau)$ and $E^{\prime}(\rho, \tau)$, but for our purposes, $E(\rho, \tau)$ is much better suited to uniform norm estimates than $E^{\prime}(\rho, \tau)$. We also write $E(\rho):=\bigcup_{\tau<\infty} E(\rho, \tau)$. It is convenient to define both $E(\infty, *)$ and $E^{\prime}(\infty, *)$ to be the class $E$ of all entire functions, and to define $E(t, \infty)=E(t)$. If we define the set-valued functions $f_{1}(\rho, \tau)=E(\rho, \tau)$ and $f_{2}(\rho, \tau)=E^{\prime}(\rho, \tau)$ for all allowable pairs $(\rho, \tau)$, then $f_{1}$ and $f_{2}$ are increasing functions in the sense that if $\left(\rho^{\prime}, \tau^{\prime}\right) \prec(\rho, \tau)$ then $f_{i}\left(\rho^{\prime}, \tau^{\prime}\right) \prec f_{i}(\rho, \tau), i=1,2$.

2.2. Hardy and Bergman spaces. We denote by $H^{p}, 0<p<\infty$, the well-known Hardy space of functions analytic in $\mathbb{D}$ for which

$$
\|f\|_{H^{p}}^{p}:=\sup _{0<r<1}\left(\int_{0}^{2 \pi}\left|f\left(r e^{i \theta}\right)\right|^{p} \frac{d \theta}{2 \pi}\right)^{1 / p}<\infty .
$$

$H^{p}$ functions have radial limits $f\left(e^{i \theta}\right)$ almost everywhere on $\mathbb{T}$. The Bergman space $A^{p}, 0<p<\infty$, is the space of functions analytic on $\mathbb{D}$ with

$$
\|f\|_{A^{p}}^{p}:=\int_{\mathbb{D}}|f(z)|^{p} d A(z)<\infty .
$$

The spaces $H^{p}$ and $A^{p}$ are Banach spaces if $1 \leq p<\infty$.

The theory of Bergman spaces, including many recent developments, can be found in $[\mathbf{H K Z}]$ and $[\mathbf{D S}]$. We mention two facts that we shall need. First

$$
(1-|z|)^{2}|f(z)|^{p} \leq\|f\|_{A^{p}}^{p}, \quad f \in A^{p}, z \in \mathbb{D},
$$

as can be seen by applying the area version of the sub-mean value property to the subharmonic function $|f|^{p}$ on $D(z, 1-|z|)$. Secondly, there exists $C=C(p)$ such that $\|f\|_{A^{p}} \leq C\|f\|_{H^{p / 2}}$; this follows from [D, Theorem 9.4].

2.3. Besov, Dirichlet, and Bloch spaces. The Besov space $B^{p}, 1<p<\infty$, is the Banach space of functions analytic on $\mathbb{D}$ for which

$$
\|f\|_{B^{p}}:=|f(0)|+\left((p-1) \int_{\mathbb{D}}\left|f^{\prime}(z)\right|^{p}\left(1-|z|^{2}\right)^{p-2} d A(z)\right)^{1 / p}<\infty .
$$

The Dirichlet space is $\mathcal{D}:=B^{2}$. The Bloch space $\mathcal{B}$ is the Banach space of all functions analytic in $\mathbb{D}$ for which

$$
\|f\|_{\mathcal{B}}:=|f(0)|+\sup _{z \in \mathbb{D}}\left(1-|z|^{2}\right)\left|f^{\prime}(z)\right|<\infty .
$$

The little Bloch space $\mathcal{B}_{0}$ is the set of all $f \in \mathcal{B}$ such that $\lim _{|z| \rightarrow 1^{-}}\left(1-|z|^{2}\right)\left|f^{\prime}(z)\right|=$ 0 . It is the natural limit as $p \rightarrow \infty$ of $B^{p}$, as well as the closure of the polynomials in $\mathcal{B}$. Note that $\mathcal{D} \subset H^{p}, 0<p<\infty$ (see [D, Chapter 6, Exercise 7]), so functions in $\mathcal{D}$ have radial limits almost everywhere on $\mathbb{T}$. For more on these spaces, see for instance $[\mathbf{Z 1}]$ and $[\mathbf{Z 2}]$.

We shall need the functions

$$
f_{r, p}:= \begin{cases}\log (1 /(1-r z))\left(\log \left(1 /\left(1-r^{2}\right)\right)^{-1 / p},\right. & 0<r<1,1<p<\infty, \\ \log ((1+r z) /(1-r z)), & 0<r<1, p=\infty .\end{cases}
$$

Then $f_{r, p} \in X^{p}$, if we define $X^{p}:=B^{p}$ when $p<\infty$, and $X^{\infty}:=\mathcal{B}_{0}$. Let $c_{r, p}:=\left\|f_{r, p}\right\|_{X^{p}}$. Then $c_{r, \infty} \leq 2$. For fixed $p<\infty, c_{r, p}^{p} \rightarrow \Gamma(p-1) /(\Gamma(p / 2))^{2}$ as $r \rightarrow 1^{-}$. Here, $\Gamma$ is the classical gamma function, and this limit can be seen by checking the proof of Theorem 1.7 in $[\mathbf{H K Z}]$ or of Lemma 4.2 .2 of $[\mathbf{Z 1}]$. In 
particular, the class of all $f_{r, p}, 0<r<1$, is a bounded subset of $X^{p}$. The functions $f_{r, 2}$ are the well-known Beurling functions, and $\left\|f_{r, 2}\right\|_{\mathcal{D}}=c_{r, 2}=1$ for all $0<r<1$.

\section{Main results}

If $1<\rho<\infty$ and $0<q<\infty$, then $E(\rho)=S\left(B^{\rho /(\rho-1)}, A^{q}\right)$; see [BV, Theorem 1]. The following theorem says that the action of $S_{\varphi}$ on a certain bounded subset $U_{R}$ of $B^{\rho /(\rho-1)}$ allows us to make finer distinctions between the classes $E(\rho, \tau)$.

Theorem 3.1. Suppose $1<\rho<\infty$ and $0<\tau, q<\infty$. Define $s:=\rho /(\rho-1)$, $R:=(2 / \tau q)^{1 / \rho}$, and $U_{R}:=\left\{c R f_{r, s}: 0<r<1, c \in \mathbb{T}\right\}$, where $f_{r, s}$ is as in (2.2). Let $F$ be the space of entire functions $\varphi$ such that $S_{\varphi}\left(U_{R}\right)$ is bounded in $A^{q}$. Then $\bigcup_{0<\tau^{\prime}<\tau} E\left(\rho, \tau^{\prime}\right) \subsetneq F \subset E(\rho, \tau)$.

Theorem 1 of $[\mathbf{B V}]$ also says that $E(1)=S\left(\mathcal{B}_{0}, A^{q}\right)$ for all $0<q<\infty$. Consequently, it is not surprising that the following analogue of Theorem 3.1 for $\rho=1$ uses a bounded subset $U_{R}$ of $\mathcal{B}_{0}$.

Theorem 3.2. Suppose $0<\tau, q<\infty$. Define $R:=2 / \tau q$, and $U_{R}:=\left\{c R f_{r, \infty}\right.$ : $0<r<1, c \in \mathbb{T}$, where $f_{r, \infty}$ is as in (2.2). Let $F$ be the space of entire functions $\varphi$ such that $S_{\varphi}\left(U_{R}\right)$ is bounded in $A^{q}$. Then $\bigcup_{0<\tau^{\prime}<\tau} E\left(1, \tau^{\prime}\right) \subsetneq F \subsetneq E(1, \tau)$.

It would be nice if we could, as in Theorem 1.1, replace the set $U_{R}$ in Theorem 3.1 by a ball in $B^{s} \cap\{f: f(0)=0\}$. This would require a variant Trudinger inequality with sharp constants, i.e. a $B^{s}$ version of the Chang-Marshall inequality. The appropriate type of variant Trudinger inequality was proven in $[\mathbf{B F V}$, Theorem 23] (see also [BO]), but not with a sharp constant. We believe that both the sharp constant problem and such a variant of Theorem 3.1 are difficult open problems. Similar remarks apply to such a variant of Theorem 3.2. However the following partial results are easy corollaries of the proofs of Theorems 3.1 and 3.2.

Corollary 3.3. Suppose $1<\rho<\infty$ and $0<\tau, q<\infty$. Define $s:=\rho /(\rho-1)$, $R:=\Gamma(s-1)^{1 / s} \Gamma(s / 2)^{-2 / s}(2 / \tau q)^{1 / \rho}$, and $K_{R}:=\left\{f \in B^{s}: f(0)=0,\|f\|_{B^{s}} \leq\right.$ $R\}$. Let $F$ be the space of entire functions $\varphi$ such that $S_{\varphi}\left(K_{R}\right)$ is bounded in $A^{q}$. Then $F \subset E(\rho, \tau)$.

Corollary 3.4. Suppose $0<\tau, q<\infty$. Define $R:=2 / \tau q$ and $K_{R}:=\{f \in$ $\left.\mathcal{B}_{0}: f(0)=0,\|f\|_{\mathcal{B}} \leq R\right\}$. Let $F$ be the space of entire functions $\varphi$ such that $S_{\varphi}\left(K_{R}\right)$ is bounded in $A^{q}$. Then $F \subset E(1, \tau)$.

Theorem 1.1 allows us to distinguish between entire functions of order two and different types, and Theorems 3.1 and 3.2 allow us to distinguish between entire functions of different orders or types, at least when one of the orders lies in $[1, \infty)$. Perhaps these assertions deserve a little justification. Suppose that $\varphi_{i}$ has order $\rho_{i}$ and type $\tau_{i}, i=1,2$, where either $\rho_{1}$ or $\rho_{2}$ lies in the interval $[1, \infty)$ and $\left(\rho_{1}, \tau_{1}\right) \prec\left(\rho_{2}, \tau_{2}\right)$. It follows that we can choose $1 \leq \rho<\infty$ and $0<\tau^{\prime}<\tau<\infty$ so that $\left(\rho_{1}, \tau_{1}\right) \prec\left(\rho, \tau^{\prime}\right) \prec(\rho, \tau) \prec\left(\rho_{2}, \tau_{2}\right)$. Then $\varphi_{1} \in E\left(\rho, \tau^{\prime}\right)$ and so $\left\|\varphi_{1} \circ f\right\|_{A^{q}}$ is uniformly bounded for all $f \in U_{R}$, where $q, R$, and $U_{R}$ are as in either Theorem 3.1 or Theorem 3.2, depending on whether $1<\rho<\infty$ or $\rho=1$. On the other hand, $\varphi_{2} \notin E(\rho, \tau)$ and so $\left\|\varphi_{2} \circ f\right\|_{A^{q}}$ is not uniformly bounded.

Let us now prove each of our theorems. 
Proof of Theorem 1.1. Suppose $\varphi \in E(2, \tau)$, and so $|\varphi(w)| \leq C \exp \left(\tau|w|^{2}\right), w \in \mathbb{C}$. Recall that $\|\cdot\|_{A^{q}} \lesssim\|\cdot\|_{H^{q / 2}}$. Thus if $f \in K_{R}$ and $g:=f / R$, then

$$
\left(\int_{\mathbb{D}}|\varphi \circ f|^{q} d A\right)^{1 / 2} \lesssim \int_{\mathbb{T}}|\varphi \circ f|^{q / 2} \lesssim \int_{\mathbb{T}} \exp \left(\frac{\tau q}{2}|f|^{2}\right)=\int_{\mathbb{T}} \exp \left(|g|^{2}\right) .
$$

But this last integral is uniformly bounded by the Chang-Marshall inequality [CM, Theorem 1] since $\|g\|_{\mathcal{D}} \leq 1$ and $g(0)=0$.

Suppose instead that $\varphi \notin E(2, \tau)$, and so there exist a sequence of nonzero points $\left(w_{n}\right)$ tending to infinity so that $\left|\varphi\left(w_{n}\right)\right| \geq n \exp \left(\tau\left|w_{n}\right|^{2}\right)$. Let $r_{n}$ be the unique number in $(0,1)$ for which

$$
\left|w_{n}\right|=R f_{r_{n}, 2}\left(r_{n}\right) \equiv R\left[\log \left(1 /\left(1-r_{n}^{2}\right)\right)\right]^{1 / 2},
$$

so that $r_{n} \rightarrow 1$ as $n \rightarrow \infty$. The Beurling functions $f_{r, 2}$ all lie in $K_{1}$, and $g_{n}:=$ $R e^{i \arg w_{n}} f_{r_{n}, 2} \in K_{R}$ has the property that $g_{n}\left(r_{n}\right)=w_{n}$. Using (2.1), we see that

$$
\begin{aligned}
\left\|\varphi \circ g_{n}\right\|_{A^{q}}^{q} & \geq\left(1-\left|r_{n}\right|\right)^{2}\left|\varphi\left(g_{n}\left(r_{n}\right)\right)\right|^{q} \\
& \geq\left(1-r_{n}\right)^{2} \cdot n \exp \left(q \tau\left|w_{n}\right|^{2}\right) \\
& =n\left(1-r_{n}\right)^{2} \exp \left(-q \tau R^{2} \log \left(1-r_{n}^{2}\right)\right) \\
& =n\left(1-r_{n}\right)^{2}\left(1-r_{n}^{2}\right)^{-2} .
\end{aligned}
$$

Since $r_{n} \rightarrow 1$, this last expression tends to infinity as $n \rightarrow \infty$. Thus $\left\|\varphi \circ g_{n}\right\|_{A^{q}}$ is not uniformly bounded.

It will actually be convenient for us to prove Theorem 3.2 first and then Theorem 3.1.

Proof of Theorem 3.2. Suppose $\varphi \in E\left(1, \tau^{\prime}\right)$ for some $\tau^{\prime}<\tau$, and so $|\varphi(w)| \leq$ $C \exp \left(\tau^{\prime}|w|\right), w \in \mathbb{C}$. Suppose also $g:=c R f_{r, \infty}$ for some $c \in \mathbb{T}, 0<r<1$. Then

$$
|\varphi(g(z))|^{q} \leq C\left|\frac{1+r z}{1-r z}\right|^{\tau^{\prime} R q} .
$$

Now $\tau^{\prime} R q=s$ for some $s<2$, and it is easily verified that $|1-r z|^{-s}$ is integrable on the unit disk, uniformly in $r$. It follows that $\varphi\left(U_{R}\right)$ is bounded is $A^{q}$.

We omit the proof that if $\varphi \notin E(1, \tau)$, then $S_{\varphi}\left(U_{R}\right)$ fails to be a bounded set, since it is very similar to the corresponding part of the proof of Theorem 1.1.

It remains to show that both containments are strict. The function $\varphi(z):=$ $\exp (\tau z)$ lies in $E(1, \tau)$. Moreover, $\varphi\left(R f_{r, \infty}(z)\right)=[(1+r z) /(1-r z)]^{2 / q}$, and it is readily verified that the $A^{q}$ norm of this last function tends to infinity as $r \rightarrow 1^{-}$. To check this, just look at the Taylor series of the function inside the modulus sign and apply the standard norm formula for the Bergman space $A^{2}$ to get

$$
\int_{\mathbb{D}}\left|\frac{1+r z}{1-r z}\right|^{2} d A(z)=1+4 \sum_{n=1}^{\infty} \frac{r^{2 n}}{n+1}
$$

The statement now follows from the monotone convergence theorem. Thus $\varphi \in$ $E(1, \tau) \backslash F$.

For each $m \in \mathbb{N}$, the function

$$
\varphi_{m}(z):=\sum_{n=m}^{\infty} \frac{\tau^{n} z^{n-m}}{n !}
$$


is entire. Clearly, $\left|\varphi_{m}(z)\right| \leq|\exp (\tau|z|)| /|z|^{m}$ for $z \neq 0$, and $\varphi_{m}(z) \gtrsim \exp (\tau z) / z^{m}$ for all sufficiently large real $z$, so $\varphi_{m} \in E(1, \tau) \backslash \bigcup_{0<\tau^{\prime}<\tau} E\left(1, \tau^{\prime}\right), m \in \mathbb{N}$.

We claim that $\left|\varphi_{m}\left(c R f_{r, \infty}(z)\right)\right|^{q}$ is integrable on $\mathbb{D}$, uniformly over all $r$ and $c$, as long as $m q>1$. Let us fix $0<r<1$, and partition $\mathbb{D}$ into annular sets $S_{j}$, $j \geq 0$, where $S_{0}:=\mathbb{D} \cap D\left(1,2\left(1-r^{2}\right)\right)$, and inductively,

$$
S_{j}:=\left[\mathbb{D} \cap D\left(1,2^{j+1}\left(1-r^{2}\right)\right)\right] \backslash S_{j-1}, \quad j \in \mathbb{N} .
$$

Let $j_{0}$ be the last integer for which $2^{j}\left(1-r^{2}\right)<2$, and so $S_{j}$ is empty for $j>j_{0}$. By considering separately the cases where $z$ lies in the left and right half of $\mathbb{D}$, we see that $|1-r z| \geq|1-z| / 2$. Since also $1-1 / s=1 / \rho$, we get

$$
\left|f_{r, \infty}(z)\right| \leq \log \left(\frac{4}{2^{j}\left(1-r^{2}\right)}\right), \quad z \in S_{j}, 0 \leq j \leq j_{0} .
$$

Let $g:=c R f_{r, \infty}$ for some $c \in \mathbb{D}$. For $0 \leq j \leq j_{0}$, we have

$$
I_{j}:=\int_{S_{j}}\left|\varphi_{m}(g(z))\right|^{q} d A(z) \leq\left(\log \left(\frac{4}{2^{j}\left(1-r^{2}\right)}\right)\right)^{-m q} \cdot\left(\frac{4}{2^{j}\left(1-r^{2}\right)}\right)^{q \tau R}\left|S_{j}\right| .
$$

where $\left|S_{j}\right|$ is the normalized area of $S_{j}$. Now $q \tau R=2$ and $\left|S_{j}\right| \leq 2^{2(j+1)}\left(1-r^{2}\right)^{2}$, so $I_{j} \lesssim\left(j_{1}+1-j\right)^{-m q}$. Thus $I:=\sum_{j=0}^{j_{0}} I_{j}$ is dominated by a bounded multiple of the series $\sum_{i=n}^{\infty} n^{-m q}$, which converges for $m q>1$, and so our claim follows. Thus $\varphi_{m} \in F \backslash \bigcup_{0<\tau^{\prime}<\tau} E\left(1, \tau^{\prime}\right)$ whenever $m$ is an integer exceeding $1 / q$.

Proof of Theorem 3.1. Suppose $\varphi \in E\left(\rho, \tau^{\prime}\right)$ for some $\tau^{\prime}<\tau$, and so $|\varphi(w)| \leq$ $C \exp \left(\tau^{\prime}|w|^{\rho}\right), w \in \mathbb{C}$. Let us fix $0<r<1$, and define $S_{j}, j \geq 0$, and $j_{0}$ as in the last part of the proof of Theorem 3.2. Noting that $1-1 / s=1 / \rho$, and arguing as before, we get

$$
\left|f_{r, s}(z)\right| \leq\left[\log \left(\frac{4}{2^{j}\left(1-r^{2}\right)}\right)\right]^{1 / \rho}, \quad z \in S_{j}, 0 \leq j \leq j_{0} .
$$

Let $g:=c R f_{r, s}$ for some $c \in \mathbb{D}$. For $0 \leq j \leq j_{0}$, we have

$$
I_{j}:=\int_{S_{j}}|\varphi(g(z))|^{q} d A(z) \leq\left(\frac{4}{2^{j}\left(1-r^{2}\right)}\right)^{\tau^{\prime} q R^{\rho}}\left|S_{j}\right| .
$$

where $\left|S_{j}\right|$ is the normalized area of $S_{j}$. Since $\left|S_{j}\right| \leq 2^{2(j+1)}\left(1-r^{2}\right)^{2}$, and $\tau^{\prime} q R^{\rho}<2$, we see that $I_{j} \lesssim\left[2^{j}\left(1-r^{2}\right)\right]^{\varepsilon}$ for some $\varepsilon>0$ which is independent of $r$ and $c$. Thus $I:=\sum_{j=0}^{j_{0}} I_{j}$ is dominated by a finite geometric series with geometric factor $2^{-\varepsilon}$ and largest term less than $2^{\varepsilon}$, so $I$ converges uniformly in $r$ and $c$. Thus $\|\varphi \circ f\|_{A^{q}}$ is uniformly bounded for all $f \in U_{R}$, as required.

We omit the proof that if $\varphi \notin E(\rho, \tau)$, then $S_{\varphi}\left(U_{R}\right)$ fails to be a bounded set, since it is very similar to the corresponding part of the proof of Theorem 1.1.

It remains to exhibit a function $\varphi \in F \backslash \bigcup_{0<\tau^{\prime}<\tau} E\left(\rho, \tau^{\prime}\right)$. The function $\varphi_{m}(z):=\sum_{n=[m / \rho]+1}^{\infty} \tau^{n} z^{[\rho n]-m} / n$ ! is entire; here $[t]$ is the greatest integer not exceeding $t$. Clearly $\left|\varphi_{m}(z)\right| \leq\left|\exp \left(\tau|z|^{\rho}\right)\right| /|z|^{m}$ for all $z \neq 0$, and $\varphi_{m}(z) \gtrsim$ $\exp \left(\tau z^{\rho}\right) / z^{m+1}$ for all sufficiently large real $z$. Thus $\varphi_{m} \in E(\rho, \tau) \backslash \bigcup_{0<\tau^{\prime}<\tau} E\left(\rho, \tau^{\prime}\right)$ for all $m \in \mathbb{N}$. We leave to the reader the task of modifying the first part of this proof to verify that $z \mapsto \varphi_{m}\left(c R f_{r, s}(z)\right)$ is uniformly in $A^{q}$ as long as $q m / \tau>1$. Thus $\phi_{m} \in F \backslash \bigcup_{0<\tau^{\prime}<\tau} E\left(\rho, \tau^{\prime}\right)$ whenever $m$ is an integer exceeding $\tau / q$. 
Corollary 3.3 follows easily from the omitted half of the proof of Theorem 3.1 and the fact that the $B^{s}$ norm of $f_{r, s}$ is asymptotic to $\Gamma(s-1)^{1 / s} \Gamma(s / 2)^{-2 / s}$. Similarly, we deduce Corollary 3.4 from the omitted half of the proof of Theorem 3.2 and the fact that $\left\|f_{r, \infty}\right\|_{\mathcal{B}} \leq 2$.

Finally, we mention two things we do not know. First, we do not know if the second containment in the conclusion of Theorem 3.1 is strict. More significantly, we know of no results similar to those in this paper that distinguish between entire functions of different orders $\rho_{1}, \rho_{2} \in(0,1)$, let alone the more refined distinctions involving different types.

\section{References}

[AMV] V. Álvarez, M.A. Márquez, and D. Vukotić, Superposition operators between the Bloch space and Bergman spaces, Ark. Mat. 42 (2004), No. 2, 205-216.

[AZ] J. Appell and P.P. Zabrejko, Nonlinear Superposition Operators, Cambridge University Press, Cambridge, 1990.

[Be] A. Beurling, Études sur un problème de majoration, Thèse pour le doctorat, Almquist \& Wieksell, Upsalla, 1933.

[Boa] R.P. Boas, Jr., Entire functions, Academic Press Inc., New York, 1954.

[BFV] S.M. Buckley, J.L. Fernández, and D. Vukotić, Superposition operators on Dirichlet type spaces, Report Univ. Jyväskylä 83 (2001), 41-61. (Papers on Analysis: dedicated to Olli Martio on the occasion of his 60th Birthday, editors: J. Heinonen, T. Kilpeläinen, and P. Koskela.) Available from http://www.math.jyu.fi/research/report83.html.

[BO] S.M. Buckley and J.O'Shea, Weighted Trudinger-type inequalities, Indiana Univ. Math. J. 48 (1999), 85-114.

[BV] S.M. Buckley and D. Vukotić, Univalent interpolation in Besov spaces and superposition into Bergman spaces, preprint.

[Ca] G. Cámera, Nonlinear superposition on spaces of analytic functions, in: Harmonic analysis and operator theory (Caracas, 1994), 103-116, Contemp. Math. 189, Amer. Math. Soc., Providence, RI, 1995.

[CG] G. Cámera and J. Giménez, Nonlinear superposition operators acting on Bergman spaces, Compositio Math. 93 (1994), 23-35.

[CM] S.-Y. A. Chang and D. E. Marshall, On a sharp inequality concerning the Dirichlet integral, Amer. J. Math. 107 (1985), 1015-1033.

[D] P.L. Duren, Theory of $H^{p}$ Spaces, Academic Press, New York-London 1970. Reprint: Dover, Mineola, New York, 2000.

[DS] P. L. Duren and A. P. Schuster, Bergman Spaces, Math. Surveys and Monographs 100, American Mathematical Society, Providence, Rhode Island, 2004.

[HKZ] H. Hedenmalm, B. Korenblum, and K. Zhu, Theory of Bergman Spaces, Graduate Texts in Mathematics, Vol. 199, Springer, New York, 2000.

[Z1] K. Zhu, Operator Theory in Function Spaces, Monographs and Textbooks in Pure and Applied Mathematics 139, Marcel Dekker, New York, 1990.

[Z2] K. Zhu, Analytic Besov Spaces, J. Math. Anal. Appl. 157 (1991), 318-336.

[Zy] A. Zygmund, Trigonometric series, Vol. I, Cambridge University Press, Cambridge, 1959.

Department of Mathematics, National University of Ireland, Maynooth, Co. KilDARE, IRELAND

E-mail address: sbuckley@maths.nuim.ie

Departamento de Matemáticas, Universidad Autónoma de Madrid, 28049 Madrid, SPAIN

E-mail address: dragan.vukotic@uam.es 